$\left.\frac{27}{2}-2\right)^{3} \cdot 6 \cdot(1)$

$$
I-19^{3} .92 \quad \quad 0 R-0500-1
$$

UCID--20316

DE85 007211

\title{
FILES USED TO GENERATE FIGURES AND TABLES IN THE APPLICATION OF THE SSMRP METHODOLOGY TO THE SEISMIC RISK AT THE ZION HUCLEAR POHER PLANT
}

\author{
Laurence L. George
}

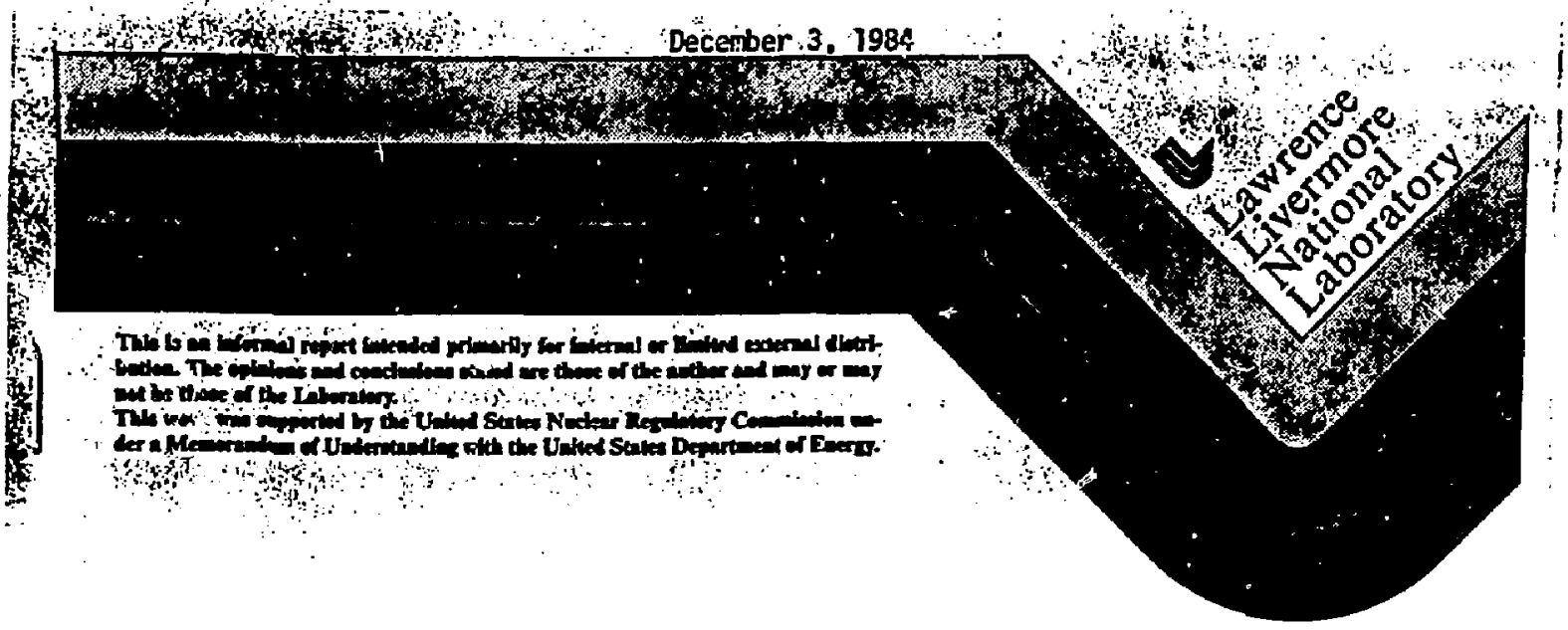




\title{
ABSTRACT
}

This report identifies some of the files used in NUREG/CR-3428. It includes microfiche copies of the files. It explains some of the differences between the files and HUREG/CR-3428.

The Seismic Safety Margins Research Program (SSMRP) was a U.S. Nuciear Regulatory Commission funded multiyear program co fucted by Lawrence Livermore National Laboratory. Its (the Program's) goal wa: to develop a complete, fully coupled analysis procedure (including method: and computer codes) for estimating the risk of an earthquake induced radioactive release from a commercial nuclear power plant. The analysis procecire was based on a stateof-the-art evaluation of then current seismic analys's and design processes. and it explicitiy attempted to account for some of the uncertainties inherent in such processes.

\section{DISCLATMRR}

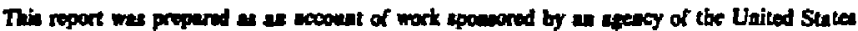

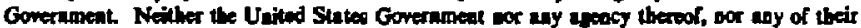

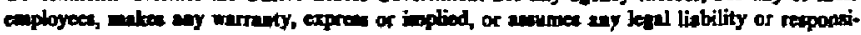

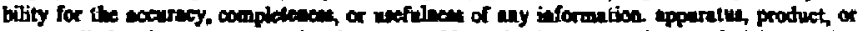

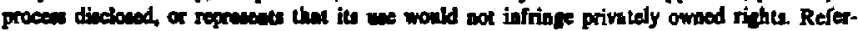
eace berein to any eppoific comenercial product, procees, or exvice by trade name, trodematk,

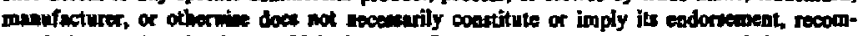
mendation, or finoriag by the Unitod Suster Government of eny agency thereof. The viewe

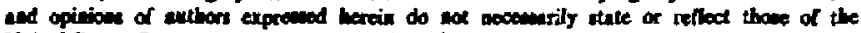
Utied State Gonernant or auy aregy thereot.
\end{abstract}


Columns 1 and 2 list the figure and tabTe numbers in NUREG/CR-3428 (Ref. 1). Colum 3 lists a brief title of the figure or table. Colum 4 jists the name of the microfiche containing relevant files. Column 5 lists the file names. Column 6 lists notes. For example, some files differ from NUREG/CR-3428, and some file names differ from the generjc names.

Most of the files in the table are on the microfiches, although their names are not. Refer to the User Manual for SEISIM (Ref. 2) for file formats to recognize the files.

The microfiche names are directory names. Appendix $B$ is a listing of the relevant directories and the files they contain. 


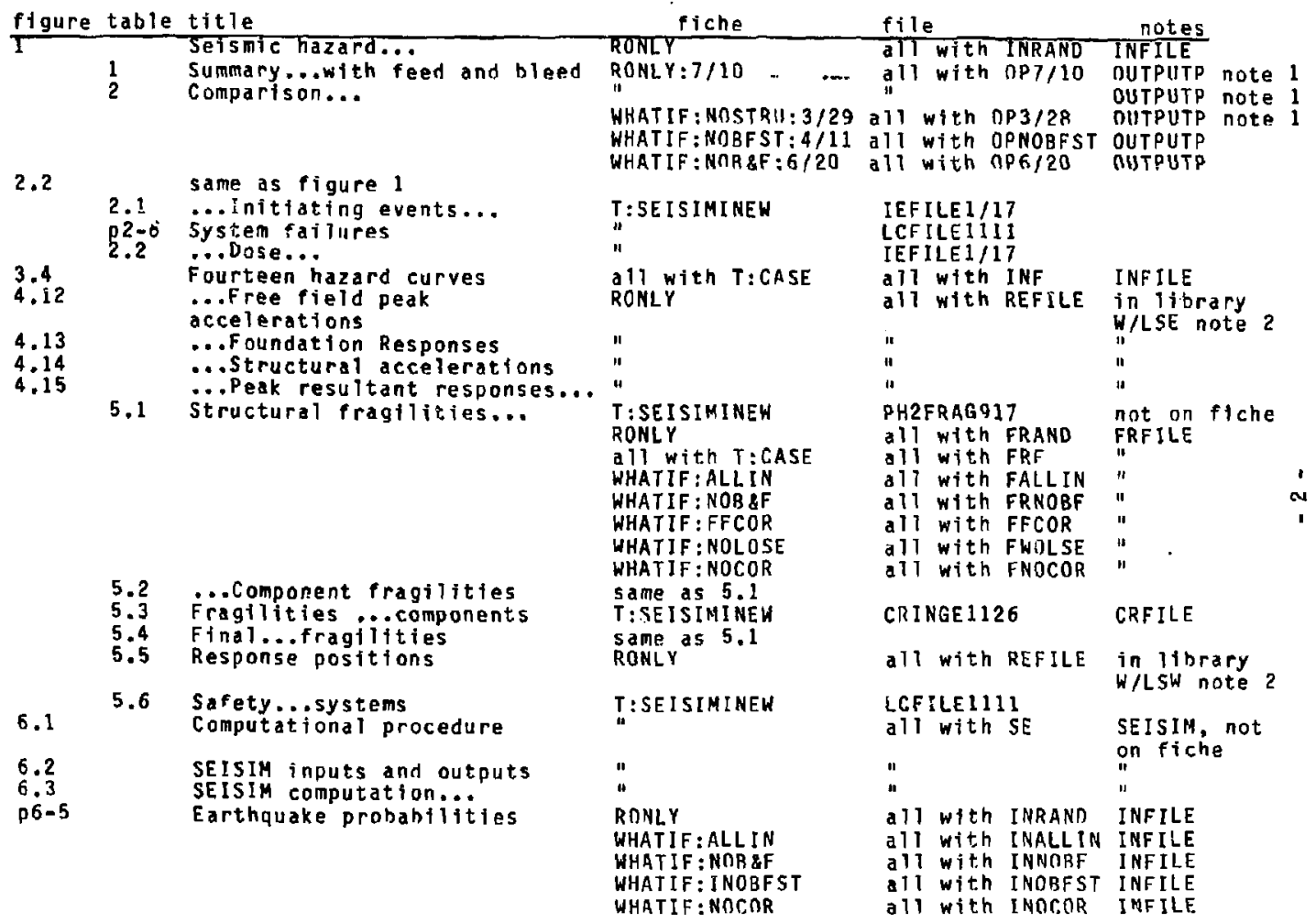


$\frac{\text { figure table titl }}{\text { pb-5 }}$

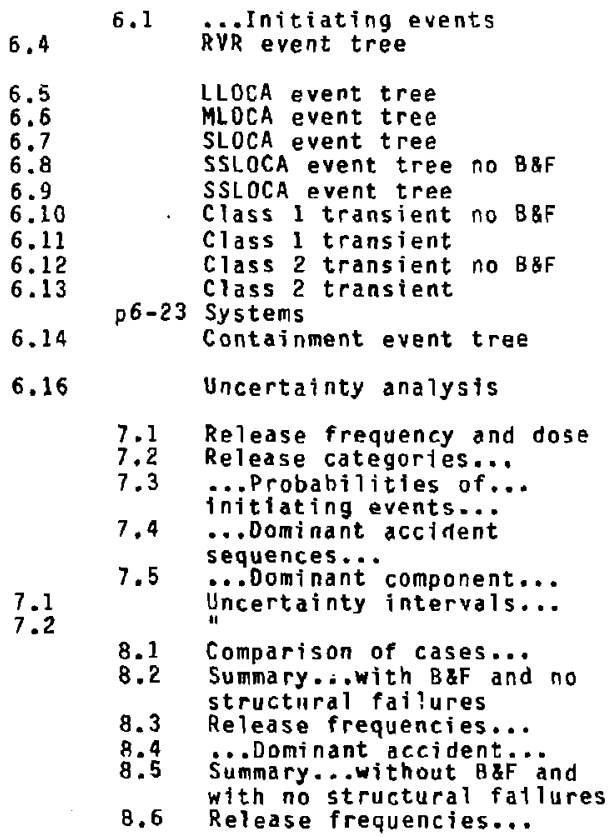

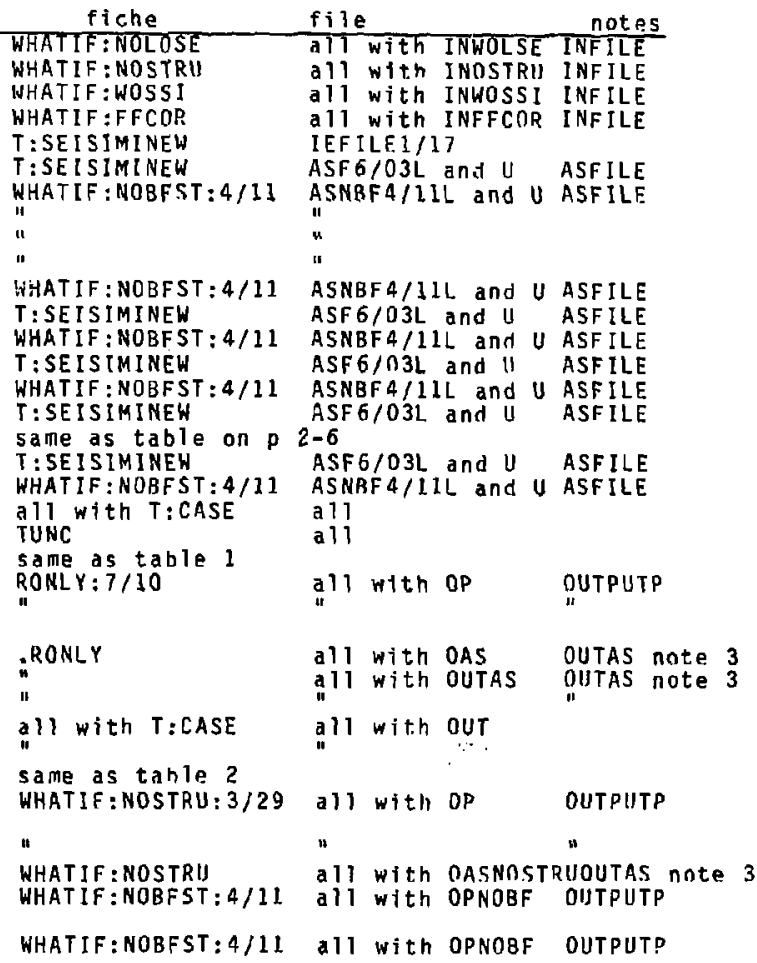




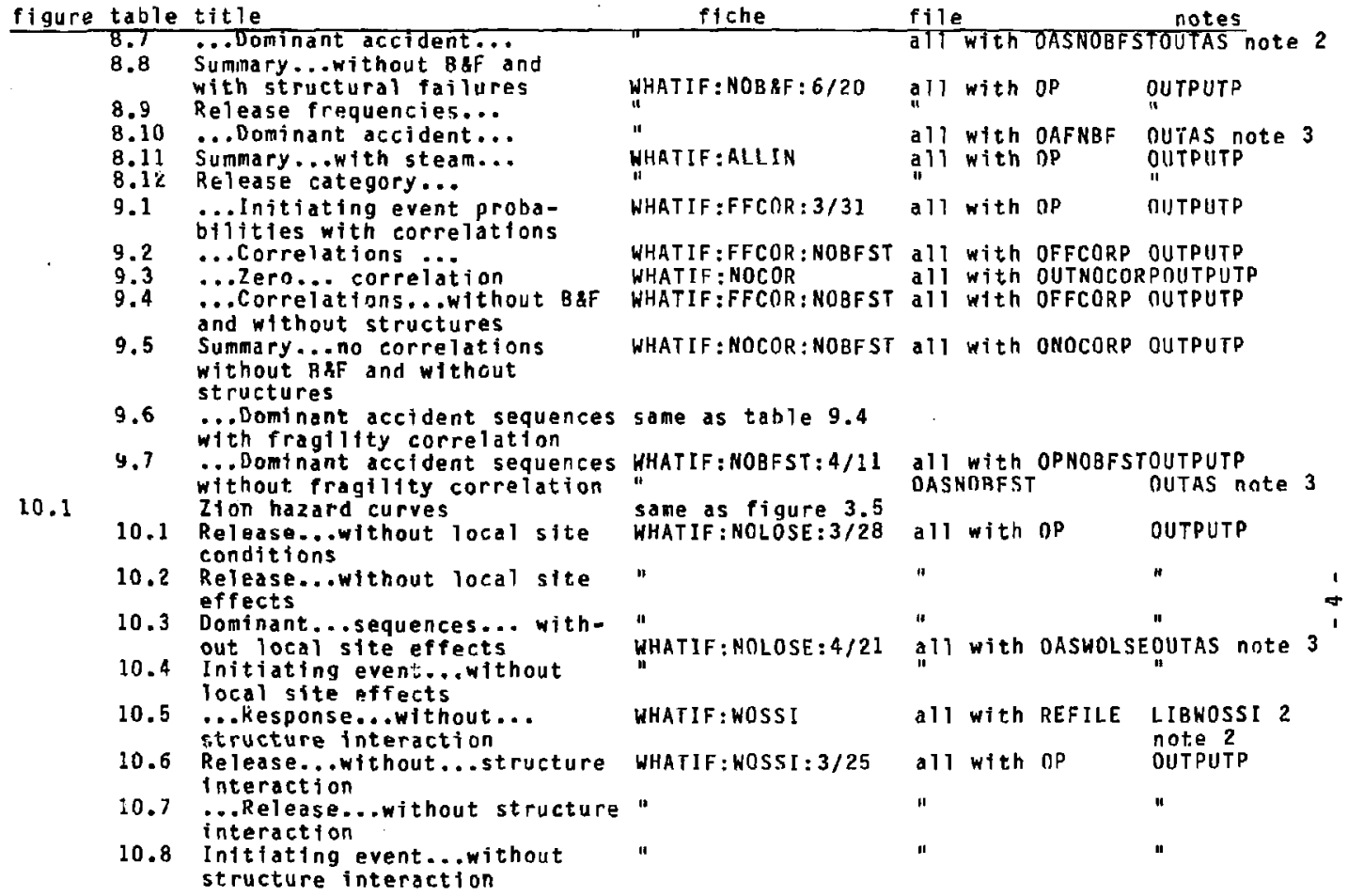




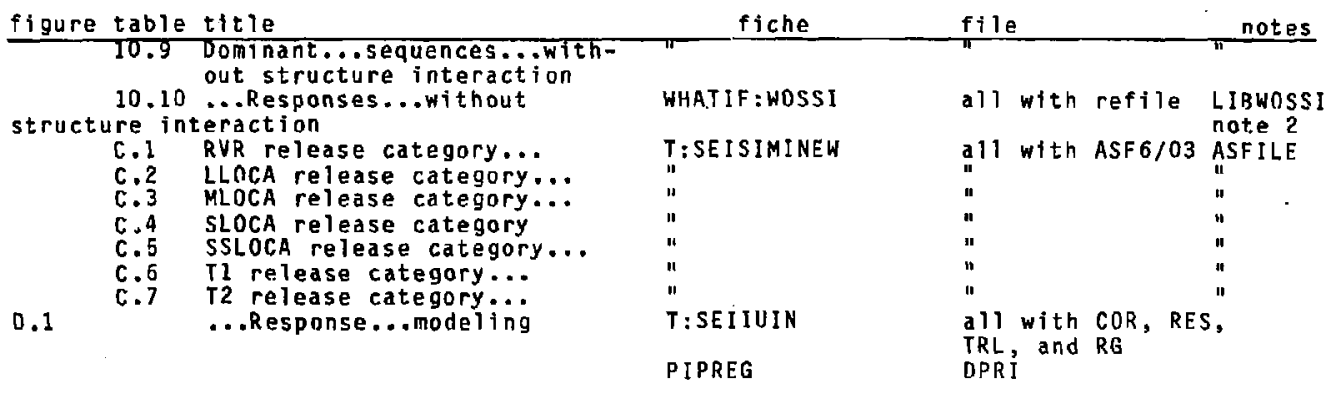


Note 1: The results in tables $7.1,7.2,7.4,8.1,8.8,8.9,8.10$, 8.11 and 8.12 are not the same as in the output files. The differences are due to some transient event sequences in file ASF $6 / 03 \mathrm{~L}$. They have had release categortes and their probabilities changed to those in file ASF 6/030. The changes occurred after the last computer run, so the changes to outputs were made by hand and reported in NUREG/CR-3428.

Failure of CSIS and CFCS(I) system is a basic event in some event sequences. According to NUREG/CR-3428 "After studying this system, studying other facilities, and discussions with personnel involved in Diablo Canyon's sefsmic study, it was decided that median fallure (sic) would occur between earthquake levels 3 and 4 of our analysis. It was therefore decided to use a step fragility function. That is, the CSIS and CFCS(I) were assumed not to fall for earthquake levels 1,2 and 3 and were assumed to fail at earthquake levels 4,5 , and $6 . . . "$ The release categarfes and their probabilities in ffles ASF6/03L and ASF6/03U are assigned accordingly.

Unfortunately some transient accfdent sequences contajn structural failures, and structural failures cause failure of CSIS and CFCS(I) regardiess of earthquake level. For those transient sequences, the release categarles in tables $C .6$ and $c .7$ for earthquake leveis 4,5 and 6 are used instead of the categories for levels 1,2 and 3 . If the release category assignments in tables C. 6 and C. 7 are applied to change the computer outputs, the results in the tables are obtained.

Note 2: Some files are stored in libraries. The library name is in the appendix. The library name and file name are in the table.

Note 3: These sensitivity analysis files are cited because they are in the microfiches. They were not used in the tables because they were superseded by later runs which are not in the micrafiches and which were not used in preparation of the tables.

References

M. P. Bohn et al "Application of the SSMRP Methodology to the Seismic Risk at the Zion Nuclear Power Plant, NUREG/CR-3428 and UCRL-53483, Lawrence Livemore National Laboratory, Livermore, CA 94550 , Jan. 1984 .

L. L. George, E. Carpenter, and J. E. Wells, "User Manual for SEISIM", UCRL 53469 draft, Lawrence Livermore Mational Laboratory, Livermore, CA 94550 , Nov. 1983. 
BASE DIRECTORIES

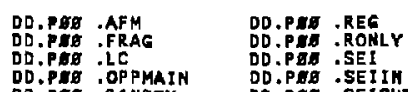

DD.PFg .OPPMAIN DD.PES .SEIIH

,REG B ENTRJES

FL, BEI DPAI

FL.PEI RLSEP

-RONLY 24 ENTRIES

\begin{tabular}{|c|c|}
\hline 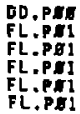 & $\begin{array}{l}\text { 7/IS } \\
\text { CRSIM } \\
\text { CUTOUT } \\
\text { FRANDI } \\
\text { FRANDZ } \\
\text { FRAND }\end{array}$ \\
\hline
\end{tabular}
SEI 27 ENTRIES

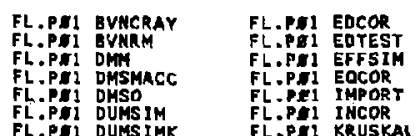

FL.PGI DUMSIMK

FL.PEI KRUSKAL

.SEIIN 18 ENTRIES

\begin{tabular}{|c|c|}
\hline 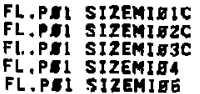 & 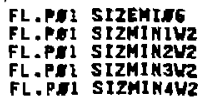 \\
\hline
\end{tabular}
.SEIOUT 1 Eg ENTRIES

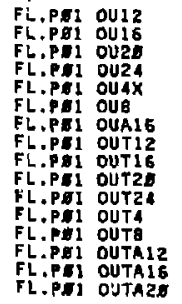

FL.PAI OUTPUTLE FL.PEl OUTPUT15 FL.PE1 OUTPUT17 FL.PEL OUTPUTIB FL.PEl OUTPUT19 FL.PEI OUTPUT2E FL.PA1 OUTPUT22 FL.PE1 OUTPUT23 FL.PE1 OUTPUT2A FL.PE1 OUTPUTS FL.PEI OUTPUTA FL,PE1 OUTPUT5 FL.PEI OUTPUTE FL.P. OUTPUT FL.PEI OUTPUTE FL.PEI OUTPUTY
DD.PES . SHIRLEY DD.PES .SIMPLE OD.PAS . T

DO.PEg :TEMP

DD.PES .TUNC
OD.PEg . WHATIF

DD.PEB . WOO

\section{FL.PEI FRSIHA FL.PEI FASIMS FL.P. FRSIME FL.PBI INRAHDI FL.PBI INRAHDI FL.PYL INRARDZ}

\section{FL.P1 KRUTEST FL.PEI MUIM FL.PEI MUSIMJK FL.PEI SEII2/4 FL.PO SEI FL.PEI SEISIMg}

\section{FL.PEI SIZMINEHZ FL.Pfi sIzMINGWZ FL.Pg1 SNXINEI}

FL.PQS SWXIHES
FL.PAJ SHXINE3

FL.PE1 OUTPUTAZ2 FL.PEI OUTPUTA23 FL+PEI DUTPUTAZ4 FL.PII DUTPUTA3 FL.P.1 OUTPUTAA FL.PEI OUTPUTA5 FL.P. I OUTPUTAG FL.PE1 OUTPUTAT FL.PEI OUTPUTAB FL P. 1 OUTPUTA LL.P OUTPU LI FL.P DUTPUTB1. FI.P. OUTPUTBII Fi.Po DUTPUTB FL.PE1 OUTPUTB 14
FL.POI OUTPUTE
FL,PEI INRAMDA
FL.PQI INRANDS
FL.PEI INRAHDG
FL,PEI RONLYSUM
FL.PEI SZCMBRANOL FL.PEI WILSE

FL.PEI SEISIMG
FL.PEI SEISIHNEW
FL,PEI SEISIMI
FL.PEI SEISIN2
FL.PEI SEP
FL.PEI SPRMANH

FL.PEI SMXINES FL.PE! SHXINES FL,PEI SMXINEG

FL.PYI OUTPUTC12 FL.PEI OUTPUTCI3 FL.PEI OUTPUTCIA FL.PAI OUTPUTCI5 FL.PEI OUTPUTC1? FL.PEI OUTPUTC1日 FL.PEI OUTPUTC19 FL.PBI OUTPUTCR2 FL.PEL OUTPUTC23 FL.PEI OUTPUTC3 FL.PEI OUTPUTCE FL:PEI OUTPUTCE FL.PRI OUTPUTC FL.PQ1 DUTPUTCG FL.PAI OUTRR

FL.PEI OUTTI 


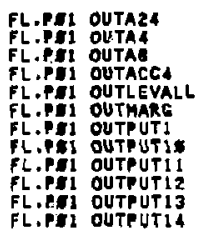

FL.PE! DUTPUTAI FL.PEI OUTPUTAIE FL.PEI OUTPUTAII FL.PEI OUTPUTAI? FL.Pfl outPuTA FL PE OUTPUTA FL.P. OUTPTAI4 FL.PI OUTPUTAIE FL.PI OUTPUTAIG FL.PI OUTPUTAIT FL.PEI OUTPUTAI. FL.PDI DUTPUTAIg FL.PAi DUTPuTAZE

.$T$

3A ENTRIES

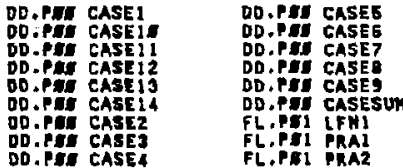

-TEMP

7. EMTRIES

\begin{tabular}{|c|c|c|c|}
\hline 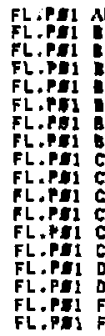 & 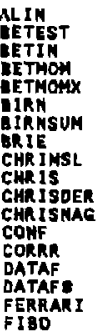 & 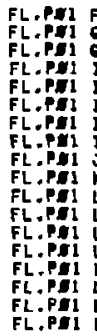 & 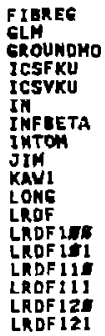 \\
\hline
\end{tabular}

- TUNC 57 ENTRIES

\begin{tabular}{|c|c|c|c|}
\hline 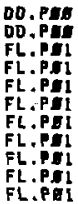 & $\begin{array}{l}3 / 25 \\
\text { A/21 } \\
\text { FREALZ } \\
\text { FUNC1 } \\
\text { FUNC2 } \\
\text { FUNC3 } \\
\text { FUNC4 } \\
\text { FUNCE } \\
\text { FUNC6 } \\
\text { FWOLSE1 } \\
\text { LHUNCI }\end{array}$ & $\begin{array}{l}F L, P E 1 \\
F L . P E I \\
F L . P E I \\
F L, P E 1 \\
F L . P E I \\
F L . P E L \\
F L . P E 1 \\
F L, P F 1 \\
F L . P E 1 \\
F L . P E I \\
F L . P E L\end{array}$ & $\begin{array}{l}\text { INUNCG } \\
\text { OASREALZ } \\
\text { OLONG } \\
\text { QUT } \\
\text { OUTASUNCI } \\
\text { OUTASUHCZ } \\
\text { OUTASUNC3 } \\
\text { OUTASUHCA } \\
\text { QUTASUHC5 } \\
\text { OUTASUHCE } \\
\text { DUTREAL2 }\end{array}$ \\
\hline
\end{tabular}

FL.PEI OUTEUTBLI? FL.PEI OUTPUTBIB FL.PEI OUTPUTB 19 FL.PE1 OUTPUTB22 FL.PEl OUTPUTB23 FL.P OUTPutas Fl.pet OUTPUTas OUTuTas FL.Pgi duTPUTB FL.PE OUTPUTBg FL.PE OUTPUTCI

FL.PAI PRAISE DD.PAS SEIIHOCONL DD.PE SEITUIH DO.PII SEISEH DO.PEI SEISIMIREY DD.PIS SEISIMOUT DD.PIS SM

DD.PAE SSMOUTNEH

FL.PII TOHS

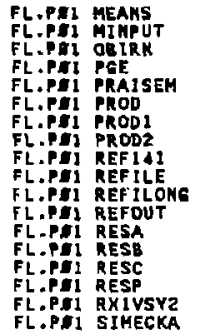

FL.PE1 OUTUNC4 FL, PE] DUTUNCS FL.PII OUTUNCS FL.PSI OUTUNCPI FL.PSt OUTUNCP EL.P FL.P\& OUTUNCPS FLP DUTUNCPS FL.PAI OUTUNCPG FL $P$. FL.PJI REAL2
FL.PQ1 OUTT12 FL.PEI DUTTIZA FL.PAL QUTT12B FL,PE1 OUTT12C FL.PEI OUTTIE FL,PEI OUTTI6A FL.PRl OUTT FL.PE OUT FL.PEI OUTTIED

FL.PEI TEAHS 1 FL.PEL TRANSX $F L+P E 1$ ZNE FL.PE1 ZAL FL.PII $2: 2$ FL.PEI ZIONL FL.PEI ZIOHZ

FL.PAI TINGLO FL.PEI TRLDNG FL.PEI TRLSPL FL.P UPLOAD EL.PAI UPLOAOS FL,PEI VROF 1ER FL-PEI VRDFLI FL.PI1 VRDF1IS FL.PEI VRDF 111 FL.PAI VRDF 12 FL.PII VRDF 121 FL.PEI WELLSHAC FL.PEI XIVSY FL.PFI XIVSY? FL.PE] XGLM

FL,PEI KTRLSPL

FL.PE1 TUNC11 FL.PEI TUNC112 FL.PGI TUNC113 FL.PEI TUMCII FL.PE TUMCI FL.PE TuNCIÍ FL.PEI TUHCIIE FL.Pal TUNCIG FL.PgI TUNCI62 FL.PE1 TUNC163 FL.PBI TUNC1GL FL.PGI TUNCIG5 


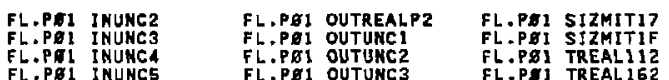

$F L, P \& I$ INUNCS

FL.PE1 OUTUNC3

$\begin{array}{ll}\text { DD.PER ALLIN } & \text { DD.PEE NOCFCS } \\ \text { DO.PEE FFCOR } & \text { DD.PER NOCOR } \\ \text { DD.PEE HOBAF } & \text { DD.PED NOLOSE } \\ \text { DD.PER HOBFST } & \text { DD.PEE NOSTRU }\end{array}$

RONLY:7/18

FL.PE1 07/18-1

FL.PEI 07/18-2

FL.PE] $07 / 18-3$

FL.PEI $07 / 18-4$

FL.PEI 07/18-5

FL.PE1 07/19-6

FL.PI OAF-IRO

FL.PAI OAF-zRO

FL.PEI OAF-3RO

FL,PEI OAF-4RO

FL.PEI OAF - SRO

FL, PEI OAF-ERO

FL.PEI OAR-IRO

FL.PEI OAR - ZRO

FL.PEI OAR-3RO

FL.PEI OAR-4RO

\section{ENTRIES}

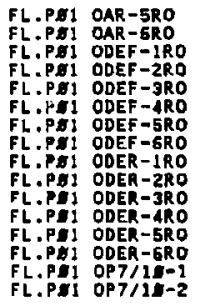

-T I CASE I

\section{ERTRIES}

FL.PEI ASFL
FL.PEI ASFU
FL.PEI CRFEL
FL.PE1 FTLNANES
FL.PE1 FRFEIL
FL.PEI FRFEIU
FL.PEI IEFILE

FL.PEI INFEII

FL.PII INF 12

FL PtI ?

FL P

FLP P

FL.PI TMFE15

FL.PEI LCFILE

-TICASE1E 14 ENTRIES

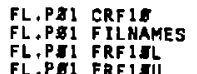

FL.PEI INF IEI

FL.PEI

FL.PEI INFIIS

.TICASEII 28 EHTRIES

\begin{tabular}{|c|c|c|}
\hline $\begin{array}{l}F L, P D 1 \\
F L, P F I \\
F L, P E 1 \\
F L, P E 1 \\
F L, P E 1 \\
F L, P D 1\end{array}$ & $\begin{array}{l}\text { ASFL } \\
\text { ASFU } \\
\text { CRFII } \\
\text { FILHAMES } \\
\text { FRFIIL } \\
\text { FRFIIU } \\
\text { IEFILE }\end{array}$ & $\begin{array}{l}F L . P E I \\
F L, P E I \\
F L . P E I \\
F L . P E I \\
F L, P E 1 \\
F L+P E I \\
F L . P E I\end{array}$ \\
\hline
\end{tabular}

.TICASE $12 \quad 37$ ENTRIES
DO, P\$E TUF

DD.P.E TUFE

DD.PEE WOSSI

FL,PE1 OP7/10-3 FL.PAI OP7/10-A FL.PDI OP7/1D-5 FL.PA FL.PEI OUTAFA-E FL.PE1 OUTAFA-6 FL.PEI OUTASA-Z FL. PEI OUTASA-3 FL.PEI OUTASA-4 FL.P\&I OUTASA-5 FL.PAI OUTASA-6 FL.PEI OUTASB-5 FL.Pe! OUTASB-G FL.P. OUTASB-6 FL.PEI OUTASUM FL.PE1 OUTCK1
FL.PEI OUTCK2
FL.PË OUTCK3 FL.PE! OUTCKA FL.PDI OUTCK5 FL.PSI OUTCKE FL.PE1 T7/16-112 FL.PDI T7/1日-112 FL.PEI T7/1\&-113 FL.PE। T7/18-114 FL.PO1 T7/18-115 FL,PGL T7/18-115 FL,PEI T7/J-16 $F L, P E 1$ T?1 FL.P. T7 T10-163 FL.P T. Th FL+Pgl T775-164 FL.PEL T7/18-165

FL.PII LUF ILE FL.PSI OSUHI1E/9 EL.PEI OUT11E/9 FL,PEI OUTA118/9 FL.PEI OUTP11E/9 FL.PE1 REFE11 FL.PEI REFEL

FL.PE1 INF1ES FL.PE1 INF I\$6 FL.PEI OSUMIE1F/9 FL.PEI OUTIEIB/9

FL.PE1 OUTA1E1E/9

FL.PE1 EUTP1EIEIG

FL,PEI LUF ILE FL.PEI OSHIIIO/1\% FL.PEI OTP IIIE/1E FL.PEI OUTIIIE/IS FL.PEI REF 111 FL.PNI REF 112

$F L, P \& 1$ REFE13 FL.PE1 REFEI FL.PEI REFO15 FL.PEI REFEI6 FL.PEI REFILE FL.PEI SEUX9/29

FL.PG 1 REF III

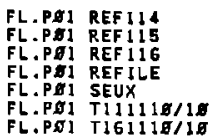




\begin{tabular}{|c|c|}
\hline 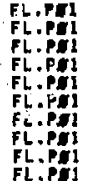 & 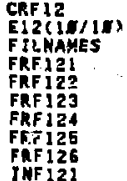 \\
\hline
\end{tabular}

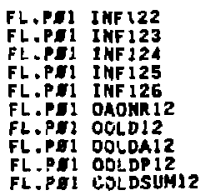

FL.PAI OONRI2

FL.PEL OPONR 12

FL.PEI OSUA121:2I

FL PII OSUMONR I?

FL PI OUTI la

FL.PEl OUTPIZIEII

FL, rNL OUTPUT

FL.PEl OUTPUTA

FL.PEL OUTPUTP

- TI cage 13 3I entr IES

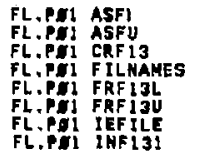

FL,PEL INF 132

FL.PEI INFI33

FL.PI INF 134

FL.PQI INF 135

FL.PEL INF 136

FL.PEL THF 136

FL.PQ LCFILE

FL.PEI OSUHI3INI

-TICAEE 14 L4 EMTRIES

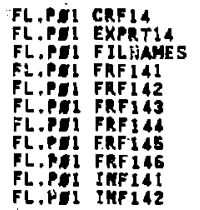

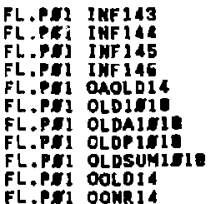

SL.PEI OQT1318/9 FLPEI CUTAIJLIE FL.PEI OUTBIJIE/O FL.PEI OUTP13IS/9 FL.PEI REF 131 FL.PE1 REF 132 PL.PE] REF 133 PL.PEI REF 134

-treasez 20 entries

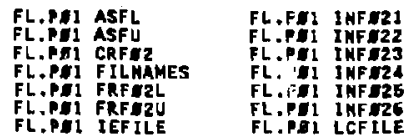

\section{.TICASE 326 EHTRIES}

\begin{tabular}{|c|c|c|}
\hline $\begin{array}{l}\text { FL. } \\
\text { FLL: } \\
\text { FLL: } \\
\text { FLL. } \\
\text { FL: } \\
\text { FL. }\end{array}$ & $\begin{array}{l}\text { ASFL } \\
\text { ASFU } \\
\text { CRFE3 } \\
\text { FILHAMES } \\
\text { FRF O3L } \\
\text { FRF } 130 \\
\text { IEFILE }\end{array}$ & $\begin{array}{l}F L . P A 1 \\
F L . P E 1 \\
F L . P E 1 \\
F L . P E 1 \\
F L . P Q 1 \\
F L . P E 1 \\
F L . P E 1\end{array}$ \\
\hline
\end{tabular}

-T:CASEA I5 ENTRIES

FL.PEI CRFE4

FL,PEI INF \& 41

FL.PEI OPOLO14 FL.PE! OPONRIA

FL PEl osuronp 14

FL. 1 OSUNONR

FL.Pei OUTdeis

RL.Pot ovTale/s

FL.pel OUTAIIIs

FL.P. OUT IE/5

FL.PAI OUTPUT

FL.Pl autPUTIE/E

\section{FL.PAI LUFILE \\ FL.PFi OSUA2IN/5 \\ FL.PE1 OUT21\%/9 \\ FL.PII OUTA $\geq 1 E / 9$ \\ FL.PII OUTA2IIIS \\ FL,PF1 OUTP218/5 \\ FL.PE1 REFE2I}

FL.PEI LUFILE

FL.PFI OSUHJIg/9

FL.PF1 OUT318/9

FL.PBI OUTPJig/9

FL.PQI REFBJI

FL,PEI INFE45
FL.PD! REFU3?

FL.PD! REF\$33

FL.PII REF 1 21 FL.PE 1 REF 122 EL.PEI REF 123 FL.PE1 REF 124 FL.PEI REF 125 FL,PEI REFI2G FL.PAI REFILE

FL.PEI REF13E

FL.PSI REF 135

FL.PAI SEUX

FL.PA1 Ti113-19/9

FL.PEl T1613-1/19

FL. PYI OUTPUTA

FL.POI OUTSUM

L.PE1 OUTSLM

FL.PE1 OUTSUMIE/E

FL.PII OUTSUMSI

FL.PE1 REF 141

FL.PSI REF 142

FL.PLI REF 143

FL,PEI REF 144

FL,P.HI REFIAE

FL.PEI REF 146

FL,PE1 REFE22

FL.P.P REF

FL.PEI REF G2

FL.POI REFM26

FL.PA1 REF ILE

FL.PAL SEUX9/29

FL.PAI REFE34

FL.PB! REFE35

FL.PBI REFE36

FL.PBI REFILE

FL.P.JI SEUXY/29

FL.Pg1 OUTALIg/g 
FL.PEL F ILHAHES FL.PEQ FRFQ4L

FL.PAI INF T42 FL.PEI INFR43 , T icAses

15 EHTRIES

FL,PEL CMFAB

FL.PGI F ILHAHES

FL,PGI FRF EGL

FL.PSI IHFESI

FL.PII INFGE2

FL.PEI INFES3

FL,PEI INFT54

-TICASEG IS ER.RIES

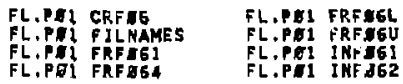

.TICASE7 74 ENTRTES

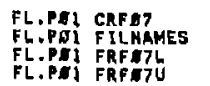

FL.PEI INF 77

FL.PEI INF

FL.PA1 IHFE73

FL.PEI FRFETU

TICASE 17 EHTAIES

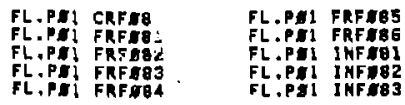

-T:CASEg 27 EHTRIES

\begin{tabular}{|c|c|c|}
\hline 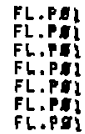 & $\begin{array}{l}\text { ASFL } \\
\text { ASFU } \\
\text { CRFA9 } \\
\text { FILNAMES } \\
\text { FRFJ9L } \\
\text { FRFJ9U } \\
\text { IEFILE }\end{array}$ & 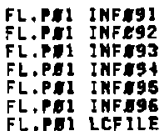 \\
\hline
\end{tabular}

G ENTR1ES

, T:CASESUM

$$
\begin{aligned}
& \text { FL.PEI CRAYSUMS } \\
& \text { FL.PEI OUT } \\
& \text { FL.PEI OUTSUHS } \\
& \text { FL.PEI SUMS }
\end{aligned}
$$

, TISEI \& UIH

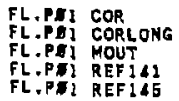

ITISEISIMINEY
FL.PEI SUMSCRAY

2E ENTRIES

FL.PEI REG 3

FL.PEI RESA

FL.PEI RESB

FL.PQ1 RESC

FL.P\&I RESCC

a6 ENTRIES
FL.PEI INFO4E

FL.PE! OSUMA18/9

FL. $r$ I OUT 1 IE/9

FL.PE! INFES5

FL.PDI INFA56

FL.PDI OSUMS1E/9

FL.PAI OUTSISIS

FL.PE1 INFE63

FL.PEI INFEG4

FL.PEI INFL65

FL.PBI INFg66

FL.PE1 INFE/5

FL.PE] 1 HF 175

FL.PEI OSUH718/9

\section{FL,PEI INFEG}

FL.PEI INFEB

FL.PEI INFEAE

FL.PE! DUTA1\%/5

FL.PEI OUTPIS/S

FL.PDI LUF ILE

FL.PII OSUM91E/9

FL.PE1 OUTS1S/9

FL,PEI OUTÁIE/9

FL.PAI OUTPG1E/9

FL.PEI OUTP916/9.PE!

FL.PEI REFEg?
FL.PEI OUTB418/9 FL.PEI OUTP418/9

FL.PEL OUTASIECS FL,PGI OUTBSIR/9 FL.PBI OUTPEID/9

FL.PEL OSUM619/9 FL,PEI OUT618/G

FL,PEI OUTPG16/9

FL.Pgl OUTAP1E/g

FL,PII OUTP7IE/S

FL.PE1 OUTPUTIE/E

FL, PEI OUTSUMIEJ

1

FL.P\$1 REF\%93

FL.POI REFEg

FL PI REF O9E

FL.PQI REF

FL,PE! SEUX

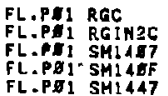

FL.PE1 SM144F

FL.PEI SOUT

FL,PQI TRL

FL.PEI TRLOHG

FL.PEI WILSESTAT 
FL.PEL ASF 6/F3L FL.P.P FL.PEI ASFIL12/10 FL.PH ASFILEI124 FL.P. ASFILEI2/3 FL.PH1 ASFILEI22A FL.PEI ASFILE3/ FL.PEI ASF ILEG/23 FL.PN1 ASTATH1/ 6 FL.PE I ASTATI1/29 FL.Pui AstaTiz/12 FL.Pel AsTATIz FL.PBI ASTATI214 FL.Pei ASTAT2/zi FL.PE1 ASTAT2/3 FL.PH ASTATA/2g FL,PEl ASTATE/6 FL.PAl AsTATG/3E FLipei astatg/B FL , PEI ASTAT7/25 FL,Pgi AsTATXEIF .T ISEISIMOUT

\section{FL,PUI EDIT2 FL.PI OU FL.P. OUT1CM1/5 FL.PE1 OUT2CH1/5 FL.PE1 OATSCM1/E FL.PH1 OUTACM1/5 FL.PE1 OUTSCM1/5}

.T I SSHOUT HEW

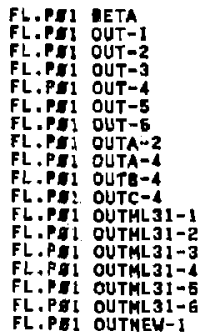

-TUNC \$3/25

FL.PEI IHUNCS FL.PF1 $03 / \div 4-1$
FL.PE1 CRINGE1/3 FL.PEI CAINGE IF/3 FL.PII CR INGE 11/ FL.PEI CRINGEI126 FL.PAI CRINEES/I9 FL.PII CRINGE 6/23 FL.PII DEAI

FL.PEI [EFILE1/17 FL.PSI IEFILE1126 EL.PA1 TEF TLE1214 FL.PAI IEF ILEg/2 FL.PEI LCFILE1III FL.PII LCFILE1213 FL.PA1 LCFILE3/22 FL.PHI LUFILEII25 FL.Pri LUF ILE121. FL.PFI LUF TLE4/2E FL,PAI LUFILEg/IB FL.Pg) OUTSUMALL FL.PFI PHIFRAG FL.PF1 PH2FRAG917 FL.PEI REFILE

\section{ENTKIES}

FL,POI OUTLEVALL FL.PE1 OUTPUT-1 FL.PE1 CUTPUT-2 FL.PE1 OUTPUT-3 FL.PEI OUTPUT-4 FL.PE1 OUTPUT-5 FL.PEl OUTPUT-E FL.PFI OUTPUTA-4

69 ENTRIES

FL.PE1 OUTMEN-2 FL.PII OUTHEY-3 FL.PA1 OUTMEU-4 FL.PII OUTMEU-5 FL.PEI DUTMEU-6 SL.PA1 OUTP-1 FL,PIS OUTP-2 FL.PEl OUTP-3 FL.PII OUTP-4 FL.P. I OUTP-5 FL.Pri OUTP-5 FL.Py OUTP-E FL.P. OUTPIE FL.P. IUTPIE-1 FL,Pg1 OUTPIE-2 FL.P. 1 DUTPIE-3 FL.PEI OUTPIE-4 FL.PE1 OUTPIE-S FL.P. OUTPIE-E

3 I ENTRIES

FL.PE1 OAS3/14-2 FL.PEI OAS3/14-3
FL.PE1 REF JLE1728 FL.PEI REFILE2728 FL.PAI REFILE3728 FL. 1 REF ILE 4728 FL.PFI REFILE5728 FL.PFI REFILE6728 FL.PEI SEIII/1\% FL.PII SEIILI7 FL.PAI SEITI/3) FL.PII SEI11/6 FL.PEI SEIIIE/S? FL.PE1 SEIIIZ17 FL.POI SEIII219 FL.PFI SEII2/17 FL.PFI SEIIZ/3 FL.PEI SEIJ3/LA FL.P. SEII3/24 FL.PEI SEIT4/5 FL.PAI SEII5/2 FL.FII SE115/14 FL.PEI SEIIT/2\% FL.PAI SEIIQU2OA

EL.PE] OUTPUTA-5 FL.PEI OUTPUTB-E FL.PEI O!ITPUTCA FL.PEI OUTPUTCM2 FL.PFI OUTPUTCM FL.PEI OUTPUTCHA FL.PEI OUTPUTCHS FL.PSI OUTPUTCHE

FL.PH1 OUTPIE FL.PSI OUTPIEICOR FL.PEI OUTPIE? FL.PFI OUTPIE FL.PFI TAPEIE-1 FL.POI TAPEIF-2 FL.PEI TAPEIO-3 FL.POI TAPEIE-4 FL.PI TAPEIO-5 FL PI TAPEIU-6 F.P. TAPEIFFL.PSI TAPEII-1 FL.PEI TAPE11-2 FL.POI TAPE11-3 FL.PEI TAPE11-4 FL.PS1 TAPE1 I-5 FL.PAI TAPE II-6 FL.PII TAPE1G-

FL.PGI TAPE16-2

FL.P.E $O P 3 / 14-4$ FL.P ${ }^{2} 1$ OP $3<14-5$ FL.PEI OP3/14-6
FL,PF] SEI โg/25

FL.PEI SEI?9/7

FL.PDI SEI IXJE/D7

FL.PEI SEI IXBE2BS

FL.PEI SEISM2 $/ 9$

FL.PDI SEISN9/24

FL,PEI SE[SNXg/24

FL.PE! SEIU日3

FL.PEI SEUA37/2!

FL.PEL SEUBJX7/21

FL.PA1 SEU9/18

FL.PB1 SEU9/2E

FL.PD 1 SEU $/ 25$

FL.PEI SEU9/29

FL.PE1 SEUX9/16

FL.PRI SEUX9/29

FL.PAI SIZEMI

FL,PEI SIZEMIRAN

FL.PEI SHXCMBMODA

FL.PEI ZIONCR 9E 7

FL,PEL OUTPUTEND FL.PII OUTPUTPIND FL.PEI SEIIXINO

FL.PEI TAPEIEIND

FL.PEI VALBLK

FL.PG1 TAPE16-3

FL.PEI TAPE16-4 FL.PSI TAPE16-6 FL.Pg1 TAPE16-6 FL.PG1 TP11:4L31-1 FL.PE1 TP1IML31-2 FL.PA1 TP1]ML31-3 FL.PE1 TPIIML31-4 FL,PG1 TP 1 ML $31-5$ FL.PEI TP $16 M L 3 L-1$ FL.PA ELPJ TPI PL.P. TP TAML31-4 FL.PEI TPIGML31-5 FL.PA] TPI GML31=6

FL.Pg1 T3/14-116 FL.Pgi T3/14-161 FL.PDI T3/14-162 
FL.PE1 $03 / 14-3$ FL.ALI $03 /\{4-4$ FL.PEI 03/14-6 FL.PA! OAS3/14-I .TUNC $+4 / 21$

FL.PE1 DASTUNC-1 FL.PB! OASTUNC-2 FL.PEI OASTURC-4 -WHATIF : ALLIN

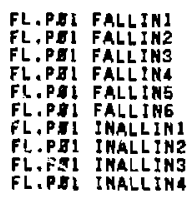

- WHATIF IFFCOR

\begin{tabular}{|c|c|}
\hline 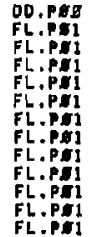 & 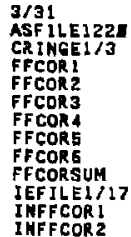 \\
\hline
\end{tabular}

- HHAT IF i NOBEF

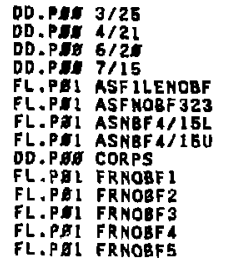

. WHATI F a MOBFST
FL,PEL OAS3/14-5 FL.PEL OAS3/14-6 FL.PB 1 QP 3/14-1 FL.PEI OP 3/14-2 FL.PEI OP $3 / 1<-3$

6 ENTRIES

FL.PEL OASTUNC-5 FL.PBI OASTUNC-S

\section{ENTRIES}

\section{FL, PEL I HALLIMS FL.PE 1 INALLIN \\ FLi? 11 O6/5-2 \\ FL.PEI 06/5-3 \\ FL.PEI O5/5-3 \\ FL.P.TI O6/5-4 \\ FLL. \\ FL.PS] $06 / 5-6$ \\ FL.PEI OAS6/5-2}

51 ENTRIES

\section{$F L+P \& 1$ INFFCOR 3}

FL.PEI INFFCORA

FL,PII INFFCOR5

FL.PEI INFFCORG

FL.PEI LCFILEIIII

FL.PHI LUFILEIZI

DOP NOBFST

FL.PEI OASFFCORI

FL.P. 1 OASFFCOR

FL.PHI OASFFCOR2

FL.PAI OASFFCOR3

FL.PGL OASFFCORA

FL,PEI OASFFCORS

\section{SS ENTRIES}

FL,PEL FRNOBF 6

FL,PEI IEFILEI2Z

FL.PEI IMNOBF 1

FLAPBI INNOBF2

$F L+P D I$ INAOEF 3

FL,PBI INHOBF4

FL.PEI IHNOBFE

FL.PE! INNOEFG

FL.PEI LCNOAF

FL.PEl LCHOBF3/23

DU, PEV OUT

FL.Pgl OUTASNOBF 1

FL.PEI OUTASNOBF Z

L.PEI OUTASHOBF 3

FL,PE1 OAS6/5-3 FL.PEI OAS6/5-4 FL.PE 1 OAS6/5-6 FL.PGI OAS6/5-G $F L+P E 1$ OPE/5-I FL PEI OPG FL

FL.PI OP6/5-

FL.PEI OP $6 / 5-6$

FL.PB! OUTFFCORI FL,PEI OUTFFCORZ FL.PEI OUTFFCOR FL.PEI OUTFFCOR FL.PGI OUTFFCOR5 FL.PUI OUTFFCORE FL.PE1 OUTFFCORP FL P I OUTFFCORP FI Fl. P4 OUTFeon .PDI OUTFFCORS FL.PEI OUTFFCORPE FL.PEI TFFCOR.111

FL,PEl TG/5-111 FL.PEI TE/5-112 FL.PGI TG/5-115 FL,PEI TG/5-116 FL.P\&I T6/5-16! FL.Pg1 T6/5-163 FLPG TS/5-i64

FL.PGI TFFCOR 112 FL.PEI TFFCORI13 FL.POI TFFCORII4 FL.PGI TFFCORII5 FL.PQ1 TFFCOR 116 FI.PG TFFCOR151 FL.PDI TFFCORIGZ FLPPI TFFCOR163 FL.PDI TFFCORIGA FL.PI T TFCORIGS FL.PEI TFFCOR166 FL.PEI W/LSE

FL.PEI OUTASNOEF 4 FL.PEL OUTAGNOBF FL.PgI OUTASNOBFG FL.PFI OUTMOBF FL.PG: QUTHOBF FL.P.1 OUTHOBF3 FL.PE! OUTMOBF $F L$.PEI DJTHOBF FL.PEL QUTHOBFS FL.PEI OUTHOBFP 1 FL.PE1 OUTNOBFPZ FL.PE1 OUTHOBFP3 FL.PGI OUTHOBFP4 FL.PEI OUTHOBFPS
FL.PEL OUTHOBFPG

FL,PEI TNOBF 111

FL.PEI TNOBFII

FL.PAL TNOBFII3

FL.PBI TNOBFII

FL.PAI THOBFIIS

FL.PE! TNOBF 116

FL,PBI TNOBF 151

FL.PEI TNOBF 162

FL,PE1 TNOEF 163

FL.PEI THOBF LEA

FL.PE】 TNOBFIES

FL.PEI THOBF 165

71 EHTRIES 
DD.PH 3/28

DD.PE

FL.PAI ASF ILEMOBF

DD.PE conPs

FL.Fil CRINGE1/3

FL,PEl FRNOEFST

FL.PEI FRMOLFST2

FL.PII FRHOAFST3

FL,PE1 FRMOIFST

FL.PE1 FRHOIFSTS

FL.PE1 FRHOAFSTS

FL.PE1 TMOHFSTI

FL.PA1 tMOAFST

$F l$, PAl I I HOLFST3

FL.PLI I IOAFSTA

FL.PA! I WOAFST

Fh.PAi I HOSFST6

FL.PEI OAGHOBFSTI

.WHAT KF, NOCOR

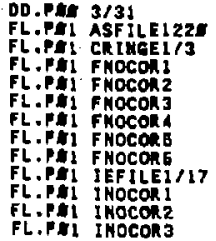

VHATIF MOLOSE

Do.pta $3 / 20$
OO.PIf $4 / 21$

FL.PI ASF ILE122

FL.PE 1 ASTATX

FL.PEI CNINGE $2 / 3$

FL,PEI FWOLSE!

FL.PE1 FWOLSE2

FL.PHI FWOLSE3

FL P T FOLS

FL.PAI FWOLSEA

FL.PEI FWOLSES

FL.PEI IEFILEIZ2E

-WHAT IF a MOSTRU

\section{DQ.PH $3 / 29$}

FL.PHI ASFILEJ22

FL.PE1 ASTATX

FL.PAI CRINGEI/3

FL.PAI CRINEEII26

FL.PEI FRHOSTRUI
FL.PEl OASHOBFST2

FL.PII OASNOEFST

FL.PII OASHOBFSTA

FL. PEI OASNOBFSTS

FL.PII OASWOBFSTE

FL.PEL ONOAFST!

FL.PEI ONOAFSTI2

FL.PEI ONOBFST?

FL.PE1 ONORFST22

FL.PQ1 ONOEFST3

F P OHOAFT32

FL. inos ont

FL.PE1 ONOAFST4

FL.Pei onoarsta

FL. PI ONOF

FL.?

FL.PE! ONOEFTS

FL.PE1 ONOBFSTE2

\section{GH ENTRIES}

FL.PII INocom 4

FL.PEI IHOcons

FL.PfI Inocoks

FL,PEI LCFILE1111

FL.PII LUFILE12I

Do.PA No.FST

fl.pel mocossur

FL.PHL OASHOCOA!

FL,PEI OASMOCON

FL,PEL OASHOCON3

FL.PII OASNOCONA

FL+PI asshocons

\section{ENTRIES}

FL, PII IHWLSE?

FL.PE1 INYOLSE2

FL.PEI IHWOLSE?

FL,PEI INWOLSE4

FL.PDI INWOLSEB

FL.PEI LCFILE1213

FL.PE1 LIALLE

FL.PII LUFILEI21E

FL,PEI OASWOLSEL

FL.PEI OASWOLSE2

FL P I OAFHOL SE

FL.PE1 DASWOLSEA

53 ENTRIES

FL.PEI I YOSTRUZ

FL.PEI INOSTRU

FL.PEI INOSTRU4

FL.PEI INOSTRUS

FL.PAI LCFILEIZI

FL.PEI LCFILEIZI3
FL,PEI LUFILEIZIE
FL.PHI OMOBFSTP 12 FL.PAI OMOBFSTP? FL,PEI OHOSFSTPZ2 FL.PAI OHOBFSTP 3 FL.PAl CHOBFSTP32 FL.PAI ONOEFSTPA FL.PA1 ONOBFSTP 42 FL.P4 OHOBFSTPE FL.PAI THOBFSTPE2 FL.P. OHDAFSTPG FL. FL. P ONOESTPE2 FL.p Thossta Fl.p Tinosfsta FL.PA TINOBFST3 FL.PT TIMOAFSTL2 FL,PEI TINOBFSTS2 FL.PH TINOBFSTE2

FL.PH T TGNOBFSTZ2

FL.PEI ouTHocos FL.P P OUTHOCOR2 FL.P. Tl OUTHOCOR FL OI OUTHOCoR FL:PI OUTHOCORA FL.PP1 OUTMOCORs F. 1 OUTHOCORG FL.P1 OUTMOCORP1 FL.Ph1 OUTHOCORP2 FL.PII OUTMOCORP3 FL.PII OUTHOCORPA FL.PHI OUTMOCORPE FL.PTI OUTWOCORPG

FL.PFI OUTKOCORP

FL.PH1 OASWOLSE 5 FL.PFi OWOLSE1

FL.PY1 OWOLSE2

FL.PAI OWOLSE3

FL.PI OYOLSE4

FL PI OWOLSES

FL

FL OWULSEP1

FL.P. OW OWOLSE3

FL.PFI OWOLSEP4

FL.PI OWOLSEPS

FL.PAI TWOLSEIII

FL.PFI OHOSTRU2 FL.P. 1 OHOSTRU3 FL.PFI OHOSTRUA FL.P. ONI ONOSTRUS FL.PEI OHOSTRU6 FL.PYI OHOSTRUP FL.PJi ONOSTRUPZ
FL.PEI TGHOBFST32

FL.PEI T6HOBFSTA2

FL.PDI T6HOBFSTS2 FL.PEI T6HOBFST62 FL.PEl TENOFBST12 Fl.pal THOBFSTL1t FL.PS1 THOBFSTI12 FL.P.I TNOBFSTI13 FL.PII TNOBFSTII FL Pgl THOBFSTI15 FL Per TMOaFsTils FL.P FL.P THOBF FL.P TN TNOBFTI62 F.P. TNOBFSTI63 FL.PE1 TNOBFSTIGA FL.PED TNOBFSTI65
FL.PNI TNOBFSTI66

FL.PEI THOCORI12 FL.PEI TNOCORIIJ Fl.P THOCON114 FL.PII TNOCOR115 FL.PEI TNOCORIIE FL.PAI THOCOR16I FL.P INOCDR16? FL.P. TH THOCDR163 FL.PAI THOCOR 164 FL.PEI THOCOR165 FL.PAI THOCÚR16G

FL.PAl TWOLSE112 FL.PJI TNOLSEI13 FL.PEI TWOLSE11 FL.PH1 TWOLSE:15 FL,PEl TWOLSE16 FL,P THOLSEIG FLP TYOL FL.P. TwoLsel63 FL.PE TWOLSE 16 PL.P. TWOLSE165 FL.PDI TWOSSI11 FL.PEI TWOSSI16

FL.PEI W/OI.SE

FL.PQ1 TNOSTRU112 FL.PEI THOSTRU:13 FL.PRI THOSTRUIIA FL.P.I THOSTRUIIS FL.PDI TNOSTRUI16 $\begin{array}{ll}F L . P Q 1 & \text { TNOSTRU161 } \\ \text { FL.P\&I TNOSTRUI62 }\end{array}$ 
FL.PEI FRNOSTRUZ FL.PEI FRNOSTRUA FL.PEI FRNOSTRU
FL.PA1 FRAOSTRUS FL.PA1 FRHOSTRUS
FL.PEI FRHOSTRUS FL.PEI IEF ILEIZZ FL.PII INOSTRU1

- VHAT IF I WOSS I

\section{DO.P $3 / 25$ \\ FL.PDE 4/2L \\ FL.PAI ASFILE12ZB \\ FL.PEI FWINGEI \\ $F L, P E !$ FWOLEE2 \\ FL. PEI FWOLSE3 \\ FL.PEI FWOLSEA \\ FL.PII FWOLSES \\ FL.PAI FWOLSE \\ FL.PEI IEFILEI22: \\ FL,PEI INYOSSII}

.WHATIF IFFCOR \& 3/31

FL.PII 03/28-1

FL.P 5 o3 $0328-2$

FL.PEI 03/28-3

FL,PEI 03:28-4

FL.PEI 03/20-5

FL.PAl 03/20-6

FL.PEl ons3/28-1

- WHATIF I FF CON I NOBF ST

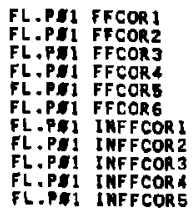

. WHAT IF \& NOBEF : $3 / 25$

FL.PEI OASKOBF-1

FL.PEI OASNOBF -2

FL.P. I OASNOBF-3

FL.PI OASMOBF-4

FL.PEI OASHOBF-5

F!.PE! OASHOBF-6

FL.PEl ONOBF - 1

FL.PEI ONOBF - 2

. WHATIF t NOBEF , 4/21
FI.PE OASNOSTRUI

FL.PEI OASNOSTRU2

FL.PgI OASNOSTAUS

FL.Pel OASHOSTRUA

FL.PEI OASHOSTRUS

FL.PEI CASHOSTRUE

FL.PEI ONOSTRUI

As ENTRIES

FL.PEI INNOSS12

FI.PEI INWOSSI3

FL.PEI INWOSSI4

FL.PQ1 INWOSSIA

FL.PQ1 INWOSSI5

FL.PEI LCFILEI213

FL.PE! LIBUOSSI

FL.PEI LUFILEIZIN

FL.PEI O2/3-3

FL.PEI $03 / 14=3$

FL.PEI OAS2/3-3

3. ENTRIEs

\section{FL.PE1 OAS3/28-3}

FL,PHI OAS3/28-4

FL.PE1 OAS3/26-5

FL.PE1 OA53/28-6

FL,PEl OP3/28-1

FL.PEI OP3/28-2

FL.PEI OP $3 / 20-3$

42 ENTRIES

FL.PEI INFFCONG

FL.PGI OASFFCORI

FL,PE1 OASFFCORE

FL.PEI OASFFCOR

FL.PSI OASFFCOR

FL. PSI DASFFCORE

FL.PII OASFFCOR

$F L, P E !$ OFFCOR 1

FL.PEI OFFCOR2

FL.PEI OFFCOR3

FL.PEI OFFCORA

\section{EHTRIES}

FL.PE! ONOBF-3 FL.PEI ONOBF-4 FL.PEI ONOBF-5 FL,PEI ONOBF-6 FL.PEI OPNOBF-1 FL PGI OPHOBF FL PEI OPHOBF-3 FL.PEI OPNOBF-4

6 ENTRIES
FL.PEI OMOSTRUP FL.PEI ONOSTRUP FL.PEI ONOSTRUPS FL.P.I I ONOSTRUPG FL.PE] OUT

FL.PGI SEIIX1219

FL.PDI TNOSTRUIII

FL.PEI DASUOSS12 FL.PGI OASWOSEIL FL.PEI OASWOSSI5 FL.PDI OP $2 / 3=3$ FL.PEI OP 3/14-3 FL.PEI CWossi? $P Q 1$ owossi FL.PEI OWOSSI5 FL.PEI DWOSSIPZ FL.PEI OWOSSIP4 FL.PEI OWOSSIP FL.PE1 T2/3-113

FL.PEL OP3/28-5 FL.PEl OP3/28-6 FL.PE1 T3/28-111 FL.PEI T3/28-112 FL.PE1 T3/28-113 FL. PE 1 T3/28-114 FL.PE1 T3/28-115 FL.PEI T3/28-116

FL.PEl OFFCOR 5 -PEI OFFCORG FL.PF1 OFFCORP 1 FL.PE1 OFFCORP 2 FL,PEI OFFCURP 3 FL.PA1 OFFCORP FL.PEI OFFCORPE FL.PE! OFFCORP 5 FL.PI TFFCOR11 FL.P T TFCOR FL.PEI TFFCOR112

FL.PEl THOSTRU163 $F L . P E I$ TNOSTRUIGA FL.PGI THOSTRUJ65 FL.PEI THESTRUIE6

FL,PDI T2/3-163 FL.PEI T3/14-113 FL.PEI T3/14-163 FL.PEI TWOSSIi12 FL.PEI TWOSSIII FL,PEI TWOSSIII4 FL,PEI TWOSSI116 FL.POL TWOSSI162

FL.PSI TWOSSII65

FL,PEL T3/28-26! L.PEI T3/2B-152 FL.PEI T3/20-I 53 FL.PEI T3/28-154 FL.PE1 T3/2B-165 FL.PAI T3/28-166

FL.PE1 TFFCOA114 FL.PE1 TFFCOR115 FL.PEI TFFCOR116 FL.PE] TFFCOR161 FL.PE1 TFFCOR162 FL.PE1 TFFCOR163 FL.PE! TFFCOR16: FL,PG] TFFCOR 165 FL.PE1 TFFCOR 165

FL.PE1 OPNOBF-5 FL.PE 1 OPNOBF-6 FL.PEI T1 I MOBF-1 FL.PAI TIIHOBF-Z FL,PEI TIINOBF-3 FL POI TINOBFFL FL.PEI TIINOBF-6

FL.PEL TIGNOBF-1

FL.PEI T1GNOBF -

FL.POI T16NOGF-3

$F L, P Q L$ TLGNOGF-4

FL.PQ1 TIGNOBF-5 


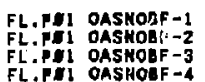

.WHATIF , HOBEF I $6 / 20$

FL.PIL O5/20-1

FL.PE] $05 / 24-2$

FL,PE1 06/2I-3

FL.PE1 06/2E-4

FL.PU1 O6/2F-5

FL.PUI 06/2N-6

FL.PEI OAFLIN-1

FL.PEI OAFLIMER-I

FL.FE1 OAFNEF-1

FL.PE1 OAFNBF-2

,WHATIF \& NOBEF I 7/15

\section{FL.PEI OAF-1MBF \\ FL.PAJ OAF-2NBF \\ FL.PE1 OAF-3HBF}

- WHAT IF , MOBAF ICONFS

\section{FL.PE: CRLAEE4/24}

FL.PT1 04/5-1

FL.PF] 04/5-2

FL.PI 04/8-3

FL.PEI O4/5-4

FL.PE! 04/5-E

Fb.PII 04/5-6

- WHAT IF I NOBEF : OUT

FL,PEI OPTE

FL.PEI OUTASNEW

FL.PEI OUTNOBIED

-WHAT IF : NOBF ST : $3 / 28$

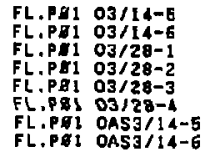

. HHAT IF : NOBF ST : 4/11

FL.PEI ASHBFA/11L

FL.PEI ASNBFilidu
FL.PEI OASNOBFSTI
FL. PE 1 OASHOBF-5

FL.PEI OASHOBF-6

40 ENTR IES

FL,Pet QAFMEF-3 FL.PII OAFNBF-4 FL.PE1 OAFKBF-5 FL.PII OAFNBF-6 FL.PEI ODEFLIM-1 FL.PES ODEFLIHER FL.PE1 ODEFHBF-1 FL.PII ODEFMBF - 2 FL.PE! ODEFHBF-3 FL.PEI ODEFGBF-4

\section{ENTRIES}

FL.PE1 OAF-ENBF FL,PEI OAF-GHBF FL.PS1 ODEF-INEF FL.PII ODEF-2HAF

3 I EHTRIES

FL. PEI OAS4/5-2 FL.PE! OAS4/5-3 FL.PI OAS4/5-4 FL. PE 1 OAS4/5-5 FL - 1 OAS4/JFL.PEI OP $4 / 5-1$ FL.PE1 OP4/5-2 FL.PEI OP 4/5-3

\section{ENTRIES}

FL.PEI OUTPUT FL.PEI OUTPUTA FL.PEl OUTPUTNEH FL.PEI OUTPUTP

\section{ENTR IES}

FL.PE1 OAS3/28-1 FL.PE1 OAS3/28-2 FL.PE1 OAS3/28-3 FL.PE1 OAS3/28-4 FL.PG) OP3/14-5 EL.PEI OP3/14-5 FL.PE1 OP3 $38-1$ FL.PQ1 OP $3 / 2 \theta-2$

31 ENTRIES

FL.PEI ONOBFSTI FL.PEI ONOBFST2
FL.PEL ODEFMBF-5 FL.PF1 ODEF MBF-6 FL.PE1 OPE/2\$-1 FL.PO1 OP 6/28-2 FL.PBI OPG $/ 2 B-3$ FL.PEI OPE/ZE-4 FL.PBI OP6/2y-5 FL.PEI OP6/2\%FL.PEI TG/28-11

FL.PE1 T6/25-112

FL.PS1 ODEF-3MBF FL.PFI ODEF-AKBF

FL.PBI ODEF-5NBF

FL.PE1 OP 4/5-4 FL.PEI OPA/E-5 FL.PS1 OP4/5-6 FL.P.1 T4/5-111 FL.PS1 TA/5-112 FL.PEI TA/5-12 FL.PBD T4/5-11 FL.PA1 TA/5-115

Fl.PE1 OUTPUTPNEU FL.PEI TAPEII FL.PE1 TAPE16

FL.P\$1 OP 3/28-3 FL.P1 OP3/28-4 FL.PII T3/14-115 FL.PEI T3/14-116 FL.PA T3/14-165 FL.P. T3/14-16 FL.PDI T3/14-15 FL.P $113 / 28-112$

FL.PE1 T4/5-116 L.PFI T46-161 FL+PE] T4/5-162 FL.PE! T4/5-163 FL.PD T4/5-164 FL.PEL TA/5-165

FL.PEI TA/5-166

FL.PE1 TAPE1GNEW

FL.PE1 T3/28-113 FL.PE1 T3/28-1L4 FL.PBl T3/20-1 FL P I I FL.P. T3/28-163

FL.PD1 T3/28-164

FL.PEI OPNOAFST3 FL.PEI OPHOBFSTA FL.PBI OPNOBFSTE 

FL.PGI OASNOBFSTA
FL.PEI OASHOBFST2 FL.PEI OASNOBFST3 FL.PD! OASHOBFST5 FL.PBI OASNOBF STG

FL.PQI ONOBFSTA FL.PEI OHOBFSTE FL.PפI ONOBFSTE FL.PEI DPNOBFST2
FL.PEI OPNOBFSTE FL. FI TINOBFST $F 1 . P E I$ TI INOBFST FL.PgI TINOBFST FL.PgI TIINOSFSTA
FL.PG1 TIGNOAFST3 FL.PE] TI 6NOBF ST FL.PG] TIGNOBFST5 FL.PQ1 T16NOBFST6
-WHAT IF I NOBFST I CORPS

\section{ENTRTES}

FL.PE: OUTAS

FL.PEI QUTPUT

FL.PEI OUTPUTP

FL.PB1 TAPEJ6

. WHATIF : HOCOR : $3 / 31$

FL.PEI $03 / 28-1$

FL.PEI 03/28-2

FL.PQ1 $03 / 28-3$

FL.PAI 03/28-6

FL.PBI OAS3/20-?

3\% ENTRIES

FL,PEI OAS $3 / 28-3$

FL.PEI OAS3/28-4

FL.PEI OAS3/2B-5

$F L, P g] O P 3 / 28-1$

$F L, P Q 1$ OP $3 / 28-2$

$F L$ PE] OP $3 / 28-3$

$F L . P E 1$ OP $3 / 28-4$

FL.PE1 OP3/28-5 FL.PQ1 $O P 3 / 28-6$ FL.P.T1 T3/28-111 $F L$.PQ1 T3/28-112 FL.PDI T3/28-113 FL.P91 T3/28-114 FL.PO1 T3/28-115 FL.PEI T3/2Q-1IE

. WHAT IF I NOCOR \& NOB F ST 43 ENTRIES

OD,PES $\{1,21$

FL.PII F NOCORL

FL.PE: FNOCOR2

$F L, P A 1$ FAOCORS

FL.PE! FNOCORE

FL.PEI INOCORI

FL.PEI INOCOR

FL.PEI I YOCOR

FL.PJi IHOCOR

- WHATIF : NOLOSE : $3 / 2 \theta$

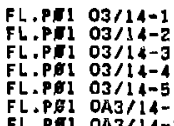

FL.PEI INOCORS

FL.PEI IMOCORE

FL.PGI OASNOCORZ

FL.PFI OASNOCORB

FL.PEI OASNOCOR5

FL.PI OASNOCORG

FL.PI OHOCOR1

Fl.per ond

FL.PI OHOCOR

26 ENTRIES

FL.PSI OA3/14-3

FL.POI OA3/14-4

FL.? $0103 / 1<-5$

FL.PQI JP $3 / 14-1$

FL.PEI OP3/14-2

FL.PGI OP 3/14-3

$F L$. PEI OP 3/14-4

FL.PEI ONOCORA

FL.PEI OHOCOR5

FL.PEI ONOCURG

FL.PEI ONOCORPI

FL.P $\& 1$ OHOCORP2

FL.PEI ONOCORP3

FL.PE1 OHOCORP5

FL.PE1 OKOCORPG

FL.PE1 THOCCR111

FL.PEI TNOCOR!12

L.PD] T3/28-161 FL.PEI T3/28-162 PL.PBI T3/28-153 FL.PDI T3/28-164 $F L, P Q 1$ T3/28-165 FL.POI T3/28-16E

FL,PE] TNOCOR:13 FL.PFI TNOCOR114 L.PEI TNOCOR11E FL.PBI TNOCOR116 $F L, P E]$ TNOCORI6! FL.PB1 TNOCOR 162 FL.PEI THOCOR163 FL.PEL TNOCOR 154 F P I TNOCOR I65

LLPI TNOCORIG

FL.PEI OP3/14-5 FL.PE1 T113/14-2 FL.PEI T113/14-3 FL.PgI T:13/14-4 FL.PEI T163/14-1

. UHATIF: NOLOSE $: 4 / 21$

5 ENTRIES

$F L$.PEI OASWOLSE-1

FL.PEI OASWOLSE-

FL.PGI OASHOLSE-3

FL.PEI DASWOLSE-4

- VHAT IF a NOSTRU : $3 / 29$

FL.PA! 09/28-1

FL.PA1 03/28-

FL.PE! 03/28-3
FL,PGL T163/14-2 FL.PQ1 T163,14-3 FL,PGI T163,14-5 FL.PBI OPNOAFST

FL.PEI 03/2B-4

$F L+P B 1$ 03/28-5

$F L, P E 1$ OAS3/20-6

FL.PMI FNOCOR

FL.PEI FHOCOR

FL.PEI OASHOCOR1

PL.PEI DASMOCORA FL,PE1 TII3/14-1
3. ENTRIES

FL,PEI DAS3/2B-3 $F L . P Q 1$
$F L . P Q S$ OAS $3 / 28-4$
FL,PणL OP 3/20-5 FL.PgI OP 3/28-6 FL.PQI T3/2G-111
FL.PB1 T3/28-161 FL.PEI T3/28-162 FL.PEI T3/28-163 


\section{FL.PE1 03/28-4 \\ FL,PE] 03/28-5 \\ FL.PL1 03/2B-6 \\ FL,PEI OAS3/2B-2}

. WHAT IF I NOSTRU $\mathrm{t} 4 / 29$

FL PEl OASHOSTR-I

FL.PEI OASHOSTR-2

FL.PI OASHOSTR-3

FL.PEI OASNOSTR-A

- WHAT IF I WOSS I $13 / 26$

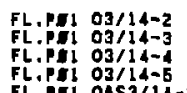

FL,P 1 OAS $3114-2$

- WHATIF, WOSS ? $1 / 2$ I

FL.PEI OAS3/28-6

FL.P\$1 OP 3/28-1

FL.PEl OP3/28-

FL.PEI OP $3 / 2 \theta-4$

FL.PH1 T3/28-112

FL.PEI T3/28-113

FL.PEI T3/2B-l14

$\begin{array}{ll}F L+P E 1 & T 3 / 28-115 \\ F L, P E S & T 3 / 28-115\end{array}$

6 ENTRIES

FL,PE2 OASHOSTR-5

2 ENTRIES

FL.PSI OAS3/14-3

FL.PII OASJ/14-4

FL.PEI OAS3/14-5

FL.PEI OF3/14-2

F-PE1 OP 3/14-4 FL.PE1 OP3/14-5 FL.PE1 T3/14-112 FL.PII T3/14-113

FL.PE1 T3/14-114
FL.PE1 T3/28-164 L.Pgl T3/28-165 FL.PEI T3/2B-166

FL,PI OASWOSS

4 ENTRIES

FL, PI: OASYOSS I-3

FL,PFi OASYOSS I-4

FL.PEI OASWOSSI- 5

- WHATIF \& MOCOR ; MOBFST, 4/21 6 EHTRIES

FL.PEI QASNOCORI FL.PEI OASNOCONE

OASNOCORz

F.PE1 T3/14-115

FL.PE1 T3/14-16a

FL.PEI T3/14-163

FL.PE1 T3/14-164

FL.PP1 T3/14-165

FL.PE1 GASNOCOR

FL,PEI OASNOCORA 